\title{
The Research of Irregularity Power Spectral Density of Beijing Subway
}

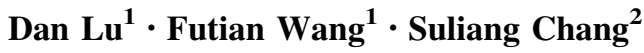

Received: 6 March 2015/Revised: 10 June 2015/ Accepted: 15 June 2015/Published online: 16 July 2015

(c) The Author(s) 2015. This article is published with open access at Springerlink.com

\begin{abstract}
This paper aimed to describe track irregularity of Beijing Subway. A preprocessing data that are from the upward track of Beijing Subway line 10 have been used in the survey. In addition, based on the maximum entropy principle, the spectrum of track irregularity power spectral density which is short for PSD has been drawn using MATLAB and fitted by using a nonlinear least squares method. After all scientific discussion, the results show the characteristic parameters of PSD. Furthermore, by contrasting Beijing Subway with national rail line, a proper function research has been done about track irregularity of Beijing Subway and the characteristics of track irregularity power spectral.
\end{abstract}

Keywords Beijing subway - Track irregularities · PSD . Fitting

Dan Lu

14125712@bjtu.edu.cn

Futian Wang

ftwang@bjtu.edu.cn

Suliang Chang

773713416@qq.com

1 State Key Laboratory of Rail Traffic Control and Safety, Beijing Jiaotong University, Beijing, China

2 The Line Branch of Beijing Subway Operation Limited Company, Beijing, China

Editor: Baoming Han

\section{Introduction}

Track irregularity is the main cause of the vibration of the locomotive vehicle and the force of the wheel rail. It has an important influence on the safety, stability, comfort, duration of vehicles and track components as well as the environmental noise of the train. When vertical profile irregularity amplitude is too large, the train will be capsized because of upward track. If the situation goes more seriously, it will cause uneven wheel load reduction or even derailing. Hence, grasp the track irregularities can monitor the track quality state, help make reasonable arrangements for the maintenance plan, reduce the costs of maintenance and repair, and ensure the safety and smoothness of the line. Master the irregularity can realize the development and application of the track quality state detection data. It can also improve the utilization rate of detection data, understand the track irregularities of the state, and help improve the reliability of the rail facilities and operating safety. It contributes to the rational allocation of resources and control maintenance costs scientifically. It has vital significance to the repair department which makes repair plans.

British Railways [1] began to study the track irregularity in 1964. Soon afterwards, Britain, Japan, East Germany, the former Soviet Union, India, Czech, the United States, and other countries drew up the spectral density and the correlation function of the respective track irregularity. In 1980s, according to six speed and track state level [2] based on the FRA Division of American Track Safety Standard, the U.S. laid down the track irregularity power spectrum.

China began the research in 1960s-1970s, Changsha Railway Institute and the Academy of Railway Sciences used the "Inertia Reference Method" [3] to measure the 
track irregularity, combined with ground testing and Inspection Car tracking and other different methods to analyze the track irregularity power spectrum. China Academy of Railway Sciences has studied on China's three main track irregularity power spectrums, and put forward the fitting function of the track irregularity power spectrum of the three main lines. There is a research [4] that makes a feature analysis of the track irregularity of urban rail transit based on track irregularity inspection data from Shanghai Subway. Xu Lei [5] analyzed the track irregularity spectrum of Shenchi-Huanghua Heavy Haul Railway, and compared it to the high- and low-interference spectrum in Germany based on raw data. It shows that there is a good correlation between TQI index and the area of track irregularity spectrum, when using MATLAB programming to analyze the power spectrum and TQI, Therefore, Jianbing $\mathrm{Li}$ [6] gave a suggestion that the track irregularity power spectrum should be one of the main factors to control the speed of the track quality.

However, due to different equipments and operation modes, the research and analysis in the National Railway are not suitable for subway. At the same time, the research about the analysis of subway is less so this paper takes the irregularity data from Beijing Subway track inspection car as sample; researching the irregularity PSD of Beijing Subway, the data in the research are from a project which is under way. Meanwhile, it makes the fitting spectrum of the track spectrum density on Beijing Subway and then gets the corresponding characteristic parameters. Thus, we can obtain a scientific evaluation and give a suitable management for railway track irregularity. In addition, we could also put forward reasonable repair advice for Beijing Subway, and provide technical support for the quality of lines.

\section{The Characteristic of Beijing Subway}

The subway is mainly responsible for the urban traffic, connecting the main passenger distribution points in a city, which makes it different from the local train and the Highspeed Rail [7].

(1) The line of Beijing Subway is underground, and its general speed can reach $30-40 \mathrm{~km} / \mathrm{h}$ while its maximum speed can run up to $80 \mathrm{~km} / \mathrm{h}$. The structure of the track is made up of heavy rail, elastic fastener, seamless lines, and monolithic track bed.

(2) Subway line is generally built in prosperous regions of the city and has to change direction frequently due to route planning, terrain, surface object, and buildings. Besides, rail wear is aggravated because the curve, especially small radius curve, occupies more and more proportion of mileage.

(3) Subway lines are in an underground and humid environment. The track structure corrosion will accelerate the destruction of the track structure because of the leakage of the tunnel.

(4) The work and repair area is narrow in the tunnel and on viaduct. Because of the high traffic density, repair and inspection work cannot be done during the daytime. Workers usually do general repair work at night.

(5) The ballast bed is laid monolithic, so there is a different characteristic. The destruction of monolithic track bed is mainly concrete crack and subsidence.

(6) Subway operation uses the ATO driving mode, which features the same type of vehicles, same axle load, and the same speed in the same section. Because of the heavy traffic and the elasticity of the monolithic roadbed, all the problems that happen in the subway would be complicated and spread fast.

\section{Test Data of Beijing Subway}

The test data are gathered from track inspection car of the Beijing Subway Company (Number: GW-01). The track inspection car is managed by testing workshop of track inspection of the Beijing Subway Company. All the measuring systems of the track inspection car, including geometric shape detection, rail full section, and the contact rail detection function, are provided by ENSCO. Types of detection data of Beijing track inspection car consist of original wave-data, overloading data, and ALD data. The existing assessment criteria of detection data on track include local gauge and track quality.

The data of track inspection car come from upstream of Beijing Subway line 10 in the situation of $40-50 \mathrm{~km} / \mathrm{h}$ and $1024 \mathrm{~m}$ length in January 4, 2014. The number of raw data is 4096 , and the measurement was taken every $0.25 \mathrm{~m}$. The raw data are stored in the binary system of g03 [8] and the data format of which is shown in Table 1.

\section{Concept and Function of Track Irregularity PSD}

Track vertical irregularity refers that the track is rutted and uneven in the vertical direction along the rail lengthwise direction. It includes roughness on the rail surface, elastic deformation of track, residual deformation, the inconsistency 
Table 1 Data format

\begin{tabular}{ll}
\hline Item & Unit \\
\hline Speed & $\mathrm{km} / \mathrm{h}$ \\
Mileage & $\mathrm{km}$ \\
Twist warp & $\mathrm{mm}$ \\
Horizontal acceleration & $\mathrm{g}$ \\
Gauge & $\mathrm{mm}$ \\
Standard & $\mathrm{mm}$ \\
Curvature & $\mathrm{Rad} / \mathrm{km}$ \\
Normal acceleration & $\mathrm{G}$ \\
Height & $\mathrm{mm}$ \\
Track & $\mathrm{mm}$ \\
Super elevation & $\mathrm{mm}$ \\
ALD & $\mathrm{v}$ \\
\hline
\end{tabular}

of components of gap and the uneven subsidence of roadbed, and so on.

The PSD diagram of track vertical irregularity is a kind of continuous curve which uses the frequency or wavelength as abscissa and the spectral density values (In unit frequency band, it means square value of the irregularity amplitude) as ordinate. It reflects the frequency distribution of the track vertical irregularity power spectrum density on the shaft of the power spectrum diagram. It can also clearly reveal each wavelength composition of random track irregularity waveform. It means that the smaller the area under the spectral line, the better the irregularity. That is to say, the lower the line locates (near the abscissa), the smoother this orbit is.

The PSD diagram of track vertical irregularity clearly indicates the wavelength components of one long rail irregularity and the density of mean square value of each wavelength inside the tiny bandwidth. Different wavelength components can give us all kinds of track irregularities information in both amplitude and wavelength ways. It plays an important role in assessing the state of the track scientifically and judging the track disease.

\section{The Calculation of Track Vertical Irregularity Power Spectrum}

The spectrum estimation of power spectral varies from theory to theory. It can be divided into the classic power spectrum estimation and modern power spectrum estimation based on the different theories. The classic power spectrum estimation is divided into direct method (periodontal method [9]) and the indirect method [10] (BT). Modern power spectrum estimation method includes parameter model [11] (AR, MA and ARMA model, Prony spectrum estimation) and non-parametric method [12] (MUSIC method, characteristic vector method).

When comparing the method of literature [13] with other methods, it is easy to see that for both the resolution and the smoothness curve, the maximum entropy spectrum estimation works best.

Maximum entropy, which is based on the known data and information, is to predict the unknown related functions of discrete time delay reasonably without any new hypothesis. A discrete information source can be represented as discrete random variables $X$, values for $x_{1}, x_{2} \ldots x_{n}$ and the values of the probability are $p_{1}, p_{2} \ldots p_{n}$. In the Shannon information theory [13], entropy is the source of each information entropy, the definition of the entropy of discrete random variable $X$ :

$H(X)=-\sum_{i=1}^{N} P\left(x_{i}\right) \ln P\left(x_{i}\right)=-E\left[\ln P\left(x_{i}\right)\right]$

In Eq. (1), the $P\left(x_{i}\right)$ is defined as the probability of the event when $X=x_{i} ; \ln P\left(x_{i}\right)$ is the definition of information. Information is needed to remove event uncertainty of measurement. $E$ stands for the mathematical expectation; while $N$ and $i$ refer to the number and serial number of event $X$. For a certain event, the probability of occurrence is 1 , so the amount of information and the corresponding entropy are zero. The smaller the probability of an event is, the greater information it has, and the bigger the corresponding entropy value is.

Use the principle of maximum entropy to do the spectral estimation:

$S(f)=\frac{P_{\mathrm{M}}}{\left|1+\sum_{m=1}^{\mathrm{M}} a_{m} e^{-j 2 \pi m \mathrm{f} T}\right|^{2}}$

In Eq. (2): $P_{\mathrm{M}}$ is the prediction error power; $m$ is the order; $N$ is signal sampling points; and $a_{m} \quad(m=1$, $2 \ldots M)$ is the coefficient of linear prediction filters of $M$.

The calculation process of track vertical irregularity power spectrum:

(1) Select 4096 data which come from track inspection car in January 4, 2014.

(2) Take a preprocessing for the selection of data, using the method of "3 Times Standard Deviation" to eliminate outliers. The outliers are data that influenced by external factor and they are beyond the normal. It will influence the analysis then.

(3) Through MATLAB programming, use the method of maximum entropy spectral estimation in the pretreatment of the raw data to draw track spectrum. 


\section{The Curve Fitting Expression of the Track Vertical Profile Irregularity Spectrum Density}

\subsection{The Fitting Function of Track Vertical Profile Irregularity Spectrum Density}

Although the curve fitting of track vertical profile irregularity spectrum density is drew under a large number of real data, it does not have any analytic function. In order to facilitate the description, it is usually expressed by a function which is close to the measured characteristic curve. Based on the fitting formula [14] of the three railway track irregularity spectrums in the U.S., Germany, and China and the analysis of the actual track inspection data, this reference documentation uses programming $\mathrm{R}$ and introduces the fitting formula of railway track irregularity power spectrum in our country:

$S(f)=\frac{A B^{2}}{f^{2}\left(f^{2}+B^{2}\right)}$

In Eq. (3), $S(f)$ refers to track irregularities fitting function; $f$ refers to spatial frequency; and $A$ and $B$ refer to spectral characteristic parameter (Table 2).

The actual fitting parameter of Beijing Subway is shown below:

Table 2 Characteristic parameter of the fitting curve of track irregularity spectrum of Beijing Subway

\begin{tabular}{lll}
\hline Characteristic parameter & $A B^{2}$ & $B^{2}$ \\
\hline Height & 883.4 & 18.69 \\
\hline
\end{tabular}

\subsection{The Fitting Theory of the Nonlinear Least Squares Method}

The fitting procedure of the nonlinear least squares method [15] is as follows: the $\left(f_{i}, S\left(f_{i}\right)\right)(i=1,2 \ldots, m)$ includes $m$ data and weight coefficient is $\omega_{0}, \omega_{1}, \ldots \omega_{m}, \mathrm{~S}\left(f_{i}, c\right)$ refers to a functional model, $c=(A, B)$ is undetermined parameter of fitting function, and $\mathrm{S}\left(f_{i}, c\right)$ is a nonlinear function whose coefficient is $c=(A, B)$, make a constructor function $s(f)$ to replace the measured curve approximately. The relationship between the constructor function curve and the measured curve is as below:

$Q=\sum_{i=0}^{m} \omega_{i}\left[S\left(f_{i}\right)-S\left(f_{i}, c\right)\right]^{2}$

In the formula (4), $Q$ refers to the quadratic sum of the distance between the two curves.

In order to get the minimum of the quadratic function, the condition must be satisfied:

$\frac{\partial Q}{\partial c_{j}}=0 \quad(j=0,1, \ldots, n)$

\subsection{The Fitting Outcome of the Track Vertical Profile Irregularity Spectrum Density Curve}

It is not difficult to see from Fig. 1 that the fitting curve trend of the Beijing Subway irregularity and the actual drawing tendency are consistent, so it can describe the irregularity state of Beijing Subway better. According to
Fig. 1 The comparison of the fitting curve between Beijing subway and national railway

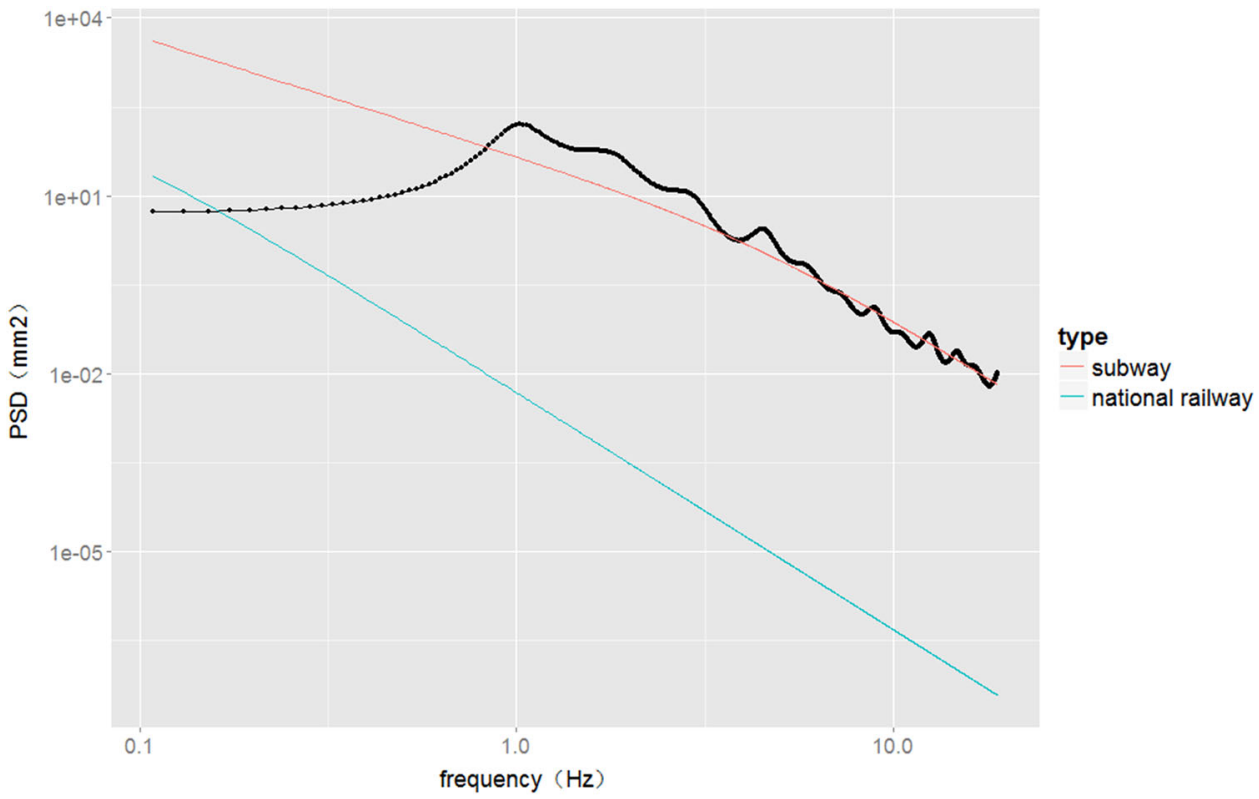


the fitting curve of Beijing Subway and national railway, Beijing Subway irregularity power spectrum curve is mostly above the national railway. The lower the curve position is, the better the track irregularity is. In this case, the national railway state is much better than subway. The frequency curve of track irregularity from 0.1 to $1.0 \mathrm{~Hz}$ is smooth. The fluctuations of the curve are more complex when it is beyond $1.0 \mathrm{~Hz}$. This is attributable to unsmooth welding joint. Subway track irregularity power spectrum frequency is obviously increased in the curve amplitude between 0.5 and $1.0 \mathrm{~Hz}$, indicating the need for better rail rolling technology and rail welding technology.

\section{Conclusions}

This paper introduces the study and significance of the track irregularity PSD. Firstly, the raw data are dealt with 3 Times Standard Deviation to eliminate outliers. Subsequently, it summarizes the operation characteristics of Beijing Subway of line 10, utilizing the maximum entropy method and MATLAB program to obtain the track spectrum estimation based on data that have been dealt, and using the nonlinear least squares method to fit the PSD curve which is proposed for Beijing Subway. Finally, comparing Beijing Subway with the national rail line, an analysis of the trend curve has been done. It points out where the track problems might have. This paper is of guiding significance to the safe operation of the subway lines and can give reasonable suggestions to the maintenance of track, so as to improve the safety of urban rail transit system. Since fitting track spectrum uses only a part of the data, the fitting formula can be a reference to the analysis of Beijing Subway track spectrum, while the fitting process needs to be further polished. At last, in order to analyze the irregularity power spectral density of Beijing Subway, it suggests that different types of data should be made full use of, and it provides more basis for managers to access the quality of orbit and master its changing rules.
Open Access This article is distributed under the terms of the Creative Commons Attribution License which permits any use, distribution, and reproduction in any medium, provided the original author(s) and the source are credited.

\section{References}

1. Liu X (2009) Research on simulation and analysis of typical track spectrum. Dissertation, Ji Lin university

2. Zeng H, Jin S, Chen X (2005) Power spectrum density analysis of track irregularity of newly-built railway line for passenger. J Railw Sci Eng 04:31-34

3. Luo L (2006) The control of track irregularity state of wheel-rail system. China Railway Publishing House, Beijing

4. Li Z (2011) Characteristic analysis of track irregularity spectrum of urban. J East China Jiaotong Univ Rail, Transit 05:83-87

5. Xu L, Chen X, Li X et al (2013) Track irregularity spectrum analysis of Shenchi-Huanghua Heavy Haul Railway. J Cent South Univ (Sci Technol) 12:5147-5153

6. Li J, Lian S (2008) Study on the relationship between track irregularity spectrum area and track quality index. J SHIJIAZHAUNG Railw Inst (Nat Sci) 04:9-12

7. Chunhua Yu (2007) The study of urban rail transit track maintenance work's contents and management mode, Urban Rail Transit, pp 72-75

8. White DL (1998) Statistical characterization of vehicle and track interaction using rail vehicle response and track geometry measurements. Dissertation, Virginia Polytechnic Institute and State University

9. Huang Y (2013) Power spectrum estimation based on periodogram. Sci Technol West China 09:1-2

10. Wang S (2011) Research on power spectrum estimation and its application in broadband ADCP signal detection. Dissertation, Ocean university of China

11. Geng X, He Z (2009) Modern digital signal processing and its application. The Press of Tsinghua University, Beijing

12. Lu M, Zhang $X$ (2005) The processing of discrete random signal. The Press of Tsinghua University, Beijing

13. Tian G (2008) The analysis and numerical simulation of railway track irregularity power spectrum. Dissertation, Southwest Jiao Tong University

14. Chen X, Wang L, Tao X et al (2008) Study on general track spectrum for Chinese main railway lines. Railw Sci Technol Res 03:73-77

15. Dong $Z$ (2005) Nonlinear least-square algorithms used for TMA in bearing-only system - the engineering mathematic model and algorithms. Inf Command Control Syst Simul Technol 01:4-8 